\title{
Aqueous Dispersions of Colloidal Boehmite: Structure, Dynamics, and Yield Stress of Rod Gels
}

\author{
Anieke Wierenga, Albert P. Philipse,* and Henk N. W. Lekkerkerker \\ Van't H off Laboratory for Physical and Colloid Chemistry, Utrecht University, Debye Research
} Institute, Padualaan 8, $3584 \mathrm{CH}$ Utrecht, The Netherlands

\author{
David V. Boger
}

Department of Chemical Engineering, University of Melbourne, Parkville, 3052 Victoria, Australia

Received April 14, 1997. In Final Form: September 8, $1997^{\circledR}$

\begin{abstract}
Thestructure, dynamics, and strength of aqueous boehmitegels werestudied by yield stress measurements and light scattering as a function of the particle aspect ratio and ionic strength. The yiel d stress was found to be a strongly varying function of the salt concentration. At low ionic strength, yield stress results from extensive double layers surrounding the rods, leading to a "colloidal glass". Addition of LiCl decreases the yield stress and enhances the partide dynamics in the gel when $[\mathrm{LiCl}]<10 \mathrm{mM}$. For $10 \mathrm{mM}<[\mathrm{LiCl}]$ $<75 \mathrm{mM}$, particle aggregation induces the formation of a space-filling heterogeneous network with fractal dimension $d=2.35$, causing a rise in theyield stress and renewed freezing of the particle dynamics. When the $\mathrm{LiCl}$ concentration exceeds $75 \mathrm{mM}$, the yield stress decreases again and the now very turbid gels show syneresis. For the attractive rods, the peculiar salt dependence of the gel strength can be explained by polydispersity in interactions, caused by inhomogeneities in particle surface chemistry and shape.
\end{abstract}

\section{Introduction}

Aqueous dispersions of charged colloidal boehmiterods at low ionic strength form self-sustaining gels at particle volumefractions bel ow $1 \%$. Thenature of thesegel s very likely differs from the gels observed in dispersions of smectic clays like laponite ${ }^{1}$ and bentonite ${ }^{2}$ because polycrystalline boehmite particles are rigid and do not swell when dispersed in water. ${ }^{3}$

Gelation of a colloidal dispersion is due to either repulsive or attractive interactions. (Here, we use the term "gel" for a colloidal dispersion with a yield stress, irrespective of the particle structure.) If the colloids are surrounded by extensive double layers, double layer overlap may strongly reduce particlediffusion, leading to the formation of a stiff repul sivegel. In an attractivegel, particles aggregate to space-filling networks, which may beeither homogeneous (dimension $d=3$ ) or heterogeneous with a lower dimension (a fractal gel). Theattractions in the network may be van der Waals forces, but for clay particles al so el ectrostaticattractions between oppositely charged surfaces may play a role, which give rise to the typical "house-of-cards" structure. ${ }^{4}$ The present work illustrates that a repulsive gel may be transformed into an attractive gel upon addition of electrolyte.

In this paper we study the relation between the structure, dynamics, and yield stress in dialyzed boehmite dispersions without added salt for particle aspect ratios

\footnotetext{
* Corresponding author.

${ }^{\otimes}$ Abstract published in Advance ACS Abstracts, November 15, 1997.

(1) Mourchid, A.; Delville, A.; Lambard, J .; Lecolier, E.; Levitz, P. Langmuir 1994, 11, 1942.

(2) Darley, H.C.H.; Gray, G. R. Composition and Properties of Drilling and Completion Fluids, 5 ed.; Gulf: Houston, 1988.

(3) Deer, W. A.; Howie, R. A.; Zussman, J. An Introduction to the Rock-Forming Minerals; Longman: New York, 1983.

(4) van Olphen, H. An Introcuction to Clay Colloid Chemistry; J ohn Wiley \& Sons: New York, 1963.
}

$r(=L / D)=9$ and $r \approx 25$. We also investigate the effect of added salt $(\mathrm{LiCl})$ on dispersion properties for rods with aspect ratio $r \approx 25$.

Boehmite dispersions are optically transparent over a large range of particle and electrolyte concentrations, allowing the study of the rod dynamics by dynamic light scattering and the use of static light scattering to investigate the particle structure. The occurrence of optically birefringent regions was inspected to detect orientational ordering in the gels.

The strength of the gel was assessed from yield stress measurements using a vane geometry as described by Nguyen and Boger .5,6 We define the yield stress, $\tau_{0}$, as the limiting amount of shear stress $(\tau)$ the particle structure within the gelled dispersion can support before it starts to flow within a reasonable experimental time scale(100-1000 s). Several authors haveraised questions about the reality of theyield stress on long timescales. ${ }^{7-9}$ In this work, the experimental yield stress is only used to quantify gel strengths. The flow behaviour on (very) long time scales is studied by low-frequency oscillatory shear measurements in the linear viscoel astic region.

Before presenting the results in section 4, we will describe experimental methods in section 3 and briefly explain therheol ogical and optical experiments in section 2. The density and mi crostructure of attractive rod gels and kinetics of rod aggregation are discussed in more detail in an accompanying paper ${ }^{10}$ hereafter referred to as I. In section 5 , we discuss the results and attempt to model the particle structure in the gels by comparing rheology and light scattering data with results from I and interaction potential calculations.

(5) Nguyen, Q. D.; Boger, D. V. J . Rheol. 1985, 29, 335

(6) Nguyen, Q. D.; Boger, D. V. J . Rheol. 1983, 27, 321

(7) Hartnett, J . P.; Hu, R. Y. Z. J . Rheol. 1989, 33, 671.

(8) Barnes, H. A.; Walters, K. Rheol. Acta 1985, 24, 323.

(9) Astarita, G. J . Rheol. 1990, 34, 275.

(10) Philipse, A. P.; Wierenga, A. M. Submitted to publication. 


\section{Theoretical Background}

2.1. The Static Structure of a Colloidal Fiber Network. Networks formed by sticky rods or fibers often have a density much lower than the density corresponding to a homogeneous random rod network, as is discussed in I. It is possible to describe a heterogeneous low-density network by so-called fractal aggregation. ${ }^{11,12}$ In I, the proportionality for the average rod volume fraction is predicted to be

$$
\Phi \frac{L}{D} \propto \frac{C}{2}\left(\frac{R_{c}}{V_{p}^{1 / 3}}\right)^{(d-3)}, \quad \text { for } \frac{L}{D} \gg 1
$$

Here $\Phi$ is the particle volume fraction of the dispersion, $L$ is the rod length, $D$ the rod diameter, $R_{c}$ the typical cluster radius, $\mathrm{V}_{\mathrm{p}}$ the particle volume, and c the average rod contact number for a homogeneous structure with a dimension $d=3$. For spheres, the dimension $d$ is known to berelated totheinteraction strengths of theaggregating particles. ${ }^{12}$ In a network of rodlike particles not only particle positions but also particle orientations may influence the dimension of a network. However, as Iong as therods (or parallel clusters of rods) in thenetwork are randomly oriented, the relation between $d$ and the aggregation kinetics as found for spheres may also apply for rod networks. ${ }^{10}$

As a result of eq 2.1, the intensity of scattered light depends on the wavenumber $k$ and the typical cluster radius $R_{c}$. When the fractal structure of the cluster is resolved, the scattered light scales with $R_{c} k$ as ${ }^{12}$

$$
I(k) \propto R_{c} k^{-d}, \text { for } 0<d<3
$$

Thefractal dimension of thenetwork is the negativeslope of the double-logarithmic plot of I (k) vs k. Note that for a homogeneous rod network ( $d=3$ ) there is no $k$ dependence of thescattered intensity, except for thesingle particle form factor, which is not included in eq 2.2.

2.2. DLS from Rods in a Gel. At shear stresses below the yiel d stress, colloi dal dispersions do not flow, because the (rotational) particle motions are severely restricted. When dispersions aretransparent, as is the casefor many boehmite gels, any residual (translational) diffusion of the rods can be measured by dynamic light scattering (DLS). As the diffusion of the rods is limited by the rigid structure, the system is effectively nonergodic. For our dispersions, the ensemble-averaged intensity autocorrelati on function for transl ational diffusion is obtained from the time-averaged intensity autocorrelation function of the small scattering volume following Pusey and Van Megen.13

2.3.1. Establishment of the Yield Stress. In this study theyield stress, $\tau_{0}$, is obtained both by extrapolation to zero shear rate of a shear flow curve and by direct measurement (thevanemethod). Theextrapolated $\tau_{0}$ may depend (strongly) on the fit equation applied. Furthermore, the occurrence of wall slip can affect the measured flow curve. Therefore, direct assessment of theyield stress in a so-called vane geometry ${ }^{5,14}$ is advantageous.

A typical example of a four-bladed vane used in our experiments is shown in Figure 1. The vane shape

(11) Family, F.; Landau, D. P. Kinetics of Aggregation and Gelation; North-Holland: Ämsterdam, 1984.

(12) Lin, M. Y.; Lindsay, H. M.; Weitz, D. A.; Ball, R. C.; Klein, R.; Meakin, P. Nature 1989, 339, 360.

(13) Pusey, P. N.; van Megen , W. Physica A 1989, 157, 705. 47.

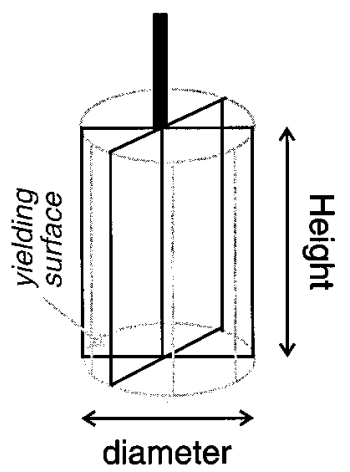

Figure 1. Schematic drawing of a vane geometry, with $I_{v}$ the length of the vane and $d_{v}$ the diameter. The "yielding surface" is indicated by the gray lines.

prevents the occurrence of wall slip. When this vane is fully immersed in a sample and the flow field can be regarded as unaffected by the container walls (large enough container), the yielding can be assumed to take placeon a surface defined by the vanedimensions (F igure 1). If the shear stress distribution along this yielding surface is homogeneous, the relation between the yield stress $\tau_{0}$ and the maximum torque $T_{m}$ on the vane can be written as

$$
\mathrm{T}_{\mathrm{m}}=\frac{\pi}{2} \mathrm{~d}_{\mathrm{v}}^{3}\left(\frac{\mathrm{I}_{\mathrm{v}}}{\mathrm{d}_{\mathrm{v}}}+\frac{1}{3}\right) \tau_{0}
$$

Here $d_{v}$ and $I_{v}$ arethe diameter and the length of thevane as denoted in Figure 1 . The rotation speed should be chosen solow that break-up of the particlestructurebefore reaching the maximum torque may be neglected. In practicethis means that therotation speed is in theregime where $\tau_{0}$ is independent of the applied shear rate. A detailed description of the vane rheometer is given by Nguyen and Boger. 5,14

2.3.2. The Linear Viscoelastic Behavior of a Yield Stress Fluid. Low-frequency oscillations in the linear viscoelastic region provide a sensitive tool to distinguish between "real" and "apparent" yield stress. When the interparticle structure is only marginally affected by the applied oscillations (linear region), a material with a "real" yield stress will show "solid-like" behavior at all frequencies $(\omega)$ lower than the molecular vibrations, with a constant storage modulus $\mathrm{G}^{\prime}$ that is much larger than the loss modulus G". However, if the dispersions only show yielding behavior on short time scales but flow at long time scales, G' will be an increasing function of $\omega$ for low frequencies to reach a constant value above a critical frequency. ${ }^{6}$

\section{Experimental Section}

3.1. Colloid Synthesis and Characterization. All measurements were performed on dispersions of boehmite rods, synthesized foll lowing Buining et al. .5,16 $^{2}$ Two dispersions of longer particles, with average lengths of approximately $180 \mathrm{~nm}$, ASP 5 and ASP 6 were prepared from a mixture of $(45.5 \mathrm{~g})$ aluminumisopropoxideand $(60 \mathrm{~g})$ aluminum-sec-butoxide in $3 \mathrm{~L}$ of a 0.085 $\mathrm{M} \mathrm{HCl}$ solution. Boehmite rods with average lengths of approximately $90 \mathrm{~nm}, \mathrm{BA} 4$ and BA6, were prepared from $150 \mathrm{~g}$ of aluminum-sec-butoxide in $3 \mathrm{~L} 0.038 \mathrm{M}$ aqueous $\mathrm{HCl}$ solution. All starting solutions were stirred for a week and then treated in an autoclave at $150^{\circ} \mathrm{C}, 4$ bar for $22 \mathrm{~h}$. After the hydrothermal

(15) Buining, P. A.; Pathmamanoharan, C.; J ansen, J . B. H.; Lekkerkerker, H. N. W. J . Am. Ceram. Soc. 1991, 74, 1303.

(16) Buining, P. A. Preparation and Properties of Dispersions of Colloidal Boehmite Rods, PhD Thesis, Utrecht University, 1992. 

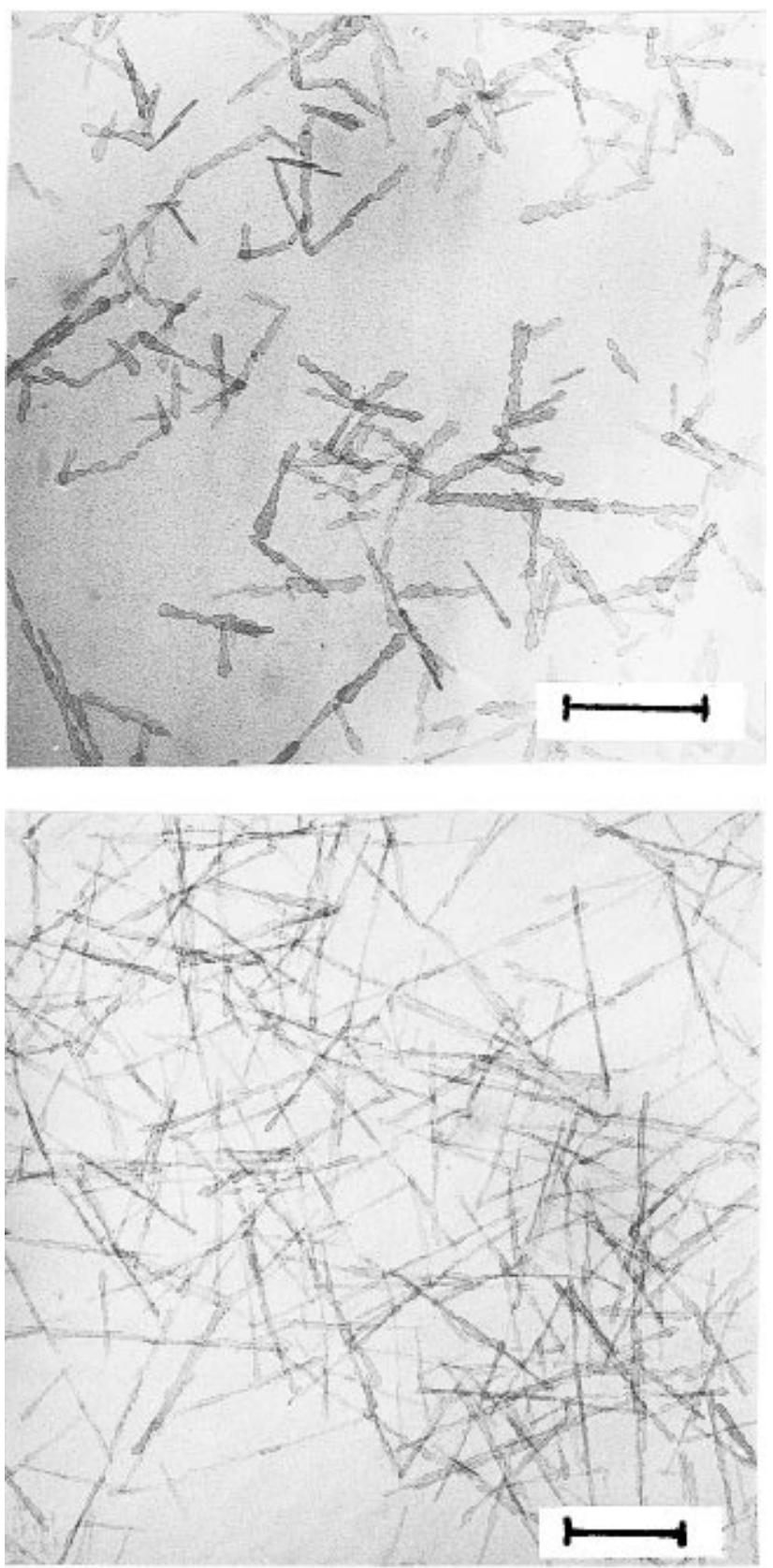

Figure 2. TEM pictures of BA4 (a, top) and ASP5 (b, bottom). The bars denote $100 \mathrm{~nm}$.

Table 1. Dimensions of the Used Rod Dispersions As Obtained from Image Analysis of the Transmission Electron Micrographs

\begin{tabular}{lccccc}
\hline system & $\begin{array}{c}\langle\text { length } \\
(\mathrm{nm})\end{array}$ & $\begin{array}{c}\text { std dev } \\
\langle\text { length } \\
(\mathrm{nm})\end{array}$ & $\begin{array}{c}\langle\text { diameter }\rangle \\
(\mathrm{nm})\end{array}$ & $\begin{array}{c}\text { std dev } \\
\langle\text { diameter } \\
(\mathrm{nm})\end{array}$ & $\langle\mathrm{L}\rangle /\langle\mathrm{D}\rangle$ \\
\hline BA4 & 85 & 24 & 9.7 & 3 & 9 \\
BA6 & 97 & 29 & 10.7 & 3.1 & 9 \\
ASP5 & 205 & 53 & 8.3 & 2 & 25 \\
ASP6 & 174 & 46 & 7.6 & 2 & 23
\end{tabular}

treatment, the dispersions were dialyzed in cellophane tubes against a continuous flow of dei onized water for 2 weeks. Typical examples of both types of rods areshown in Figure2. The particle dimensions obtained by Image Analysis of the transmission micrographs are given in Table 1.

Using el ectrophoresis, Buining ${ }^{17}$ estimated for boehmiterods a surface potential $\xi=70 \mathrm{mV}$. In view of the similar synthesis

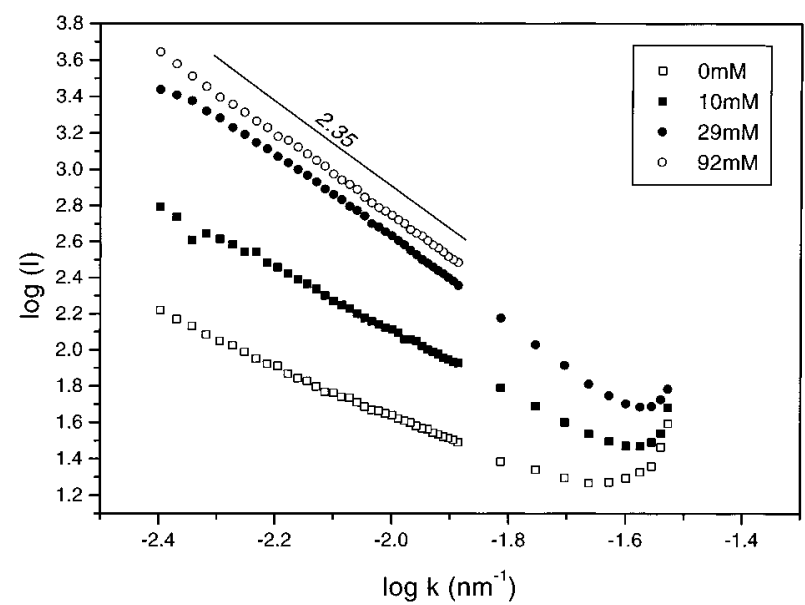

Figure 3. SLS curves for ASP 3 dispersions with $\Phi=0.33 \%$ and $[\mathrm{LiCl}]=0 \mathrm{mM}(\square),[\mathrm{LiCl}]=10 \mathrm{mM}(\boldsymbol{\square}),[\mathrm{LiCl}]=29 \mathrm{mM}$ $(\bullet)$, and $[\mathrm{LiCl}]=92 \mathrm{mM}(\mathrm{O})$. A linear dependence of $\ln (\mathrm{I})$ on $\mathrm{ln}(\mathrm{k})$ is observed for $0.0045 \mathrm{~nm}^{-1}<\mathrm{k}<0.013 \mathrm{~nm}^{-1}$.

route we assume that this value is also a reasonable estimate or the $\xi$-potential of the particles used in this work. Conductivity measurements ${ }^{18}$ indicate that the monovalent electrolyte concentration in the boehmite samples without added electrolyte may be approximated by $0.1 \Phi \mathrm{mol} / \mathrm{L}$.

3.2. Sample Preparation. In all experiments deionized water and LiCl (p.a.) wereused. Concentration of thedispersions was achieved by evaporation of the solvent in a water bath at approximately $60{ }^{\circ} \mathrm{C}$ either under a continuous $\mathrm{N}_{2}$ flow or at reduced pressure. The volume fraction of the samples was determined by drying and weighing, using the density of pure boehmite $(3.01 \mathrm{~g} / \mathrm{mL})$ as the particle density.

Thesamples for rheol ogy werehomogenized by fierceshaking. Trapped air bubbles were removed by centrifuging the samples for 10-30 $\mathrm{min}$ at $1000 \mathrm{rpm}$. The ionic strength of the ASP 5 dispersions was changed by adding a weighed amount of $\mathrm{LiCl}$ solution to a dispersion. The samples were homogenized by stirring with a spatula. Vanemeasurements on thesedispersions were carried out both immediately after preparation of the samples and after at least $12 \mathrm{~h}$ of equilibration time. For most dispersions no significant difference between the two readings was found, but in all cases theresults of the equilibrated samples wereused. Toprevent contami nation of thesamples with silicates or $\mathrm{CO}_{2}$, the samples were al ways stored in closed pl astic bottles. Only samples with a homogeneous appearance were used for rheological experiments.

For light scattering experiments, $\mathrm{LiCl}$ series of ASP6 dispersions with four different volume fractions between $0.17 \%$ and $1.15 \%$ were prepared by adding a weighed amount of a $\mathrm{LiCl}$ sol ution to a dispersion in a light scattering cuvette. Toenhance mixing, the dispersions were liquefied at $50{ }^{\circ} \mathrm{C}$ before the electrolyte solution was added. Then the two fluids weremixed thoroughly with a vortex mixer. The mixing was considered complete when the separating meniscus between added fluid and "initial" dispersion had disappeared (this sometimes required mixing for more than $14 \mathrm{~h}$ ). Air bubbles trapped in the very viscous samples were subsequently removed by centrifuging for $5 \mathrm{~min}$ at $500 \mathrm{rpm}$. All light-scattering samples were stored in a thermostated room at $21.5 \pm 0.1{ }^{\circ} \mathrm{C}$ and were inspected on flocculation, sedimentation, bi refringence, and flowing properties on a regular basis over a period of 3 months.

3.3. Static and Dynamic Light Scattering. Static light scattering (SLS) was performed with a Fica 50 photometer at a wavelength of $546 \mathrm{~nm}$ using vertical-vertical polarising filters. Typical curves are shown in Figure 3. The fractal dimension of the rod networks was determined from a linear fit through the data points for scattering angles between $15^{\circ}$ and $60^{\circ}$.

The long-time collective diffusion in the gel-like dispersions was studied with dynamiclight scattering(DLS), using a software correlator to measure autocorrelation functions over timescales 
of $10^{2} \mathrm{~s}$. The wavel ength was $674.1 \mathrm{~nm}$ (Spectra Philips Model 2020 krypton laser), and all measurements were made at a scattering angle of $90^{\circ}$ using vertical-vertical polarizing filters. The ensemble averaged scattered intensity was obtained as the average scattered intensity measured when the cuvette was rotated in the laser beam. The polarization of scattered light was negligible ( $<1 \%$ of the total scattered intensity) for the samplesthat do not show strong permanent bir efringence(hence, in this work, only for the ASP 6-dispersion with $\Phi=0.33 \%$ and no added $\mathrm{LiCl}$, the intensity of polarized light was significant). All experiments werecarried out in a thermostatic bath of $25^{\circ} \mathrm{C}$.

3.4. Rheology Measurements. Two different rheometers were used for the rheological experiments: a Haake-RV3 rheometer (vane geometry) for direct yield stress measurements and a Carri-med 100/2 controlled stress rheometer (cone-andplate geometry) for shear flow and oscillatory shear measurements.

TheH aake-RV3 was provided with a vane-geometry consisting of four thin blades (Figure 1) as described in detail by Nguyen and Boger.6,14 For rotation rates between 0.1 and $0.4 \mathrm{rpm}$, no ratedependence of $\tau_{0}$ was observed. All experiments werecarried out at a rotation speed of $0.2 \mathrm{rpm}$, which has been established as the optimal rate for yield stress measurements in colloidal gels. ${ }^{19}$ The vanes had lengths of $20-40 \mathrm{~mm}$ and diameters of $10-20 \mathrm{~mm}$. The plastic bottle used as a sample container had a diameter of $32 \mathrm{~mm}$. For each separate sample, care was taken tousethevanewith optimal dimensi ons for thesensitivity needed. Sincenosignificantly different results wereobtained using vanes with varying lengths and diameters in the same dispersions, it was assumed that wall effects did not affect the experiments. It was checked for several samples that the value of the maximum torque did not change significantly when the rotating speed of the vane was either doubled or halved.

For the Carri-med measurements three cone-and-plate geometries with different cone angles $(\theta)$ and diameters (d) were used $\left(\mathrm{I}, \mathrm{d}=2 \mathrm{~cm}, \theta=2^{\circ} ; \mathrm{II}, \mathrm{d}=4 \mathrm{~cm}, \theta=1^{\circ} 59^{\prime} ; \mathrm{II}, \mathrm{d}=6 \mathrm{~cm}\right.$, $\left.\theta=1^{\circ} 59^{\prime}\right)$. If any variation of the measured curves was found using different geometries, data obtained with the smallest geometry were used. All experiments were performed at $20^{\circ} \mathrm{C}$, using a thermostatic bath to keep the temperature constant. It was checked for all cases that the oscillatory shear experiments were carried out in the linear viscoelastic regime $\left(\gamma \leq 2 \times 10^{-4}\right.$ rad).

\section{Results}

Theresults obtained for dispersions without added salt are presented in section 4.1, and the effect of increasing the ionic strength on the gel properties of the dispersions of the highest aspect ratio rods (ASP5 and ASP6) is described in section 4.2 .

4.1. Boehmite Dispersions without Added Salt. Microstructure and Stability. When the boehmite dispersions were concentrated to particl evolumefractions above theinitial value after hydrothermal treatment and dialysis, the turbidity increased. Birefringence was observed when the dispersions ( $\Phi \geq 0.35 \%$ ) with longer particles (ASP5 and ASP6) were illuminated between crossed polarizers. Raising the particle volume fraction yielded more interference colors; the degree of orientational orderingincreased. The shorter particles (BA4 and BA6) were not found to form permanent birefringent structures in the dispersions ( $\Phi \leq 3.9 \%$ ), although flow birefringence was observed at volume fractions higher than $1 \%$.

During evaporation of the solvent, vigorous (magnetic) stirring could not prevent the formation of gel-likelumps with a much higher concentration than the continuous phase. These could be redispersed by stirring the dispersions (in closed bottles) for $12 \mathrm{~h}$ at room temperature. However, upon concentrating the ASP 5 dispersion to particle volume fractions higher than $2 \%$, irreversible

(19) Pashias, N.; Green, M. D. Personal communication.

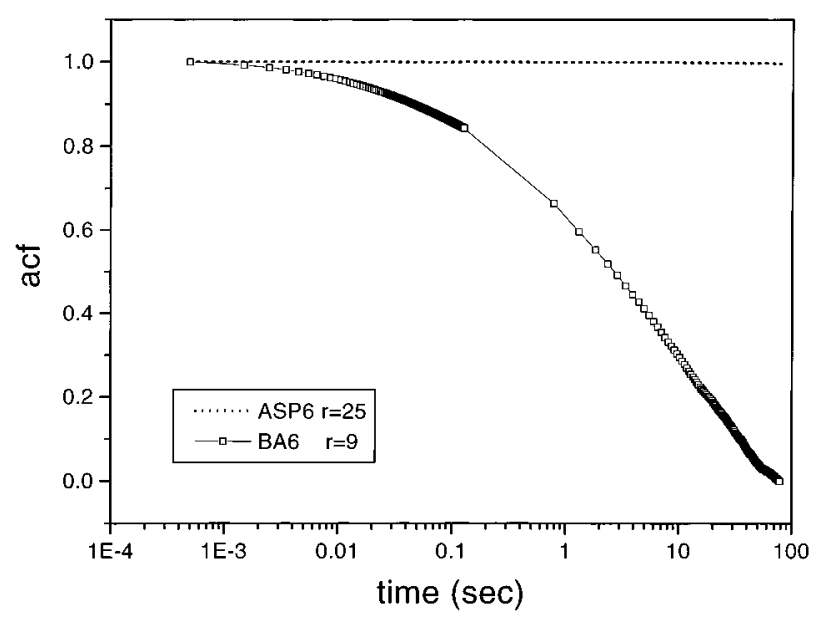

Figure4. Intensity autocorrelation function for self-sustaining gels of BA6 $(2.5 \%)$ and ASP6 $(0.33 \%)$, measured using DLS.

flocculation occurred and very inhomogeneous samples were formed without high yield stresses. Theflocculated samples were not used for further experiments.

Although in the dispersions of short particles (BA4 and BA6) such irreversible flocculation was not observed, no dispersions with particle volume fractions above $3.9 \%(\mathrm{v} /$ v) were used. When $\Phi \geq 4 \%$, the viscosity of the samples is so high that stirring the sample during evaporation becomes inefficient, and concentration inhomogeneities cannot be prevented.

Dynamic Light Scattering. In Figure 4 the autocorrelation functions are shown for a $0.33 \%$ dispersion of long rods (ASP6) and a 2.8\% dispersion of short rods (BA6). Thesedispersions are both self-sustaining and do not flow under gravity. The dynamics of the long rods seems to be completely frozen on a time scale of $80 \mathrm{~s}$. The short rods behave differently. Here the intensity autocorrelation function completely relaxes within $80 \mathrm{~s}$.

Rheology. In Figure 5, the concentration dependence of the yield stress is shown for ASP 5 with rods of aspect ratio $r=25$ and BA4 with $r=9$. The dispersions of the longer particles have a significant yield stress al ready at $\Phi=0.35 \%$ and the short rods at $\Phi=1.1 \%$. Upon concentration, the short rods are capable of building the strongest gels. The data points in Figure 5 are steady state maxima of the shear stress; in time, the torque on the vane did not change significantly after reaching this maximum.

For both ASP5 and BA4 dispersions, the yield stress values as shown in Figure 5 were measured within $24 \mathrm{~h}$ after preparing the samples. After the samples were allowed to stand for 3 weeks, the yield stress values were found to be increased by $10-50 \%$. However, when the yield stress was measured again $24 \mathrm{~h}$ after vigorous shaking, the values did not differ significantly from the values in Figure 5.

The storage moduli $\left(G^{\prime}\right)$ of the dispersions in the linear viscoelastic regime are shown in Figure 6. Only for the ASP5 dispersions with $\Phi=0.99 \%$ and $1.7 \%$ was a frequency-independent storage modulus measured for $0.001 \mathrm{~Hz} \leq \omega \leq 100 \mathrm{~Hz}$. For the other samples, $\mathrm{G}^{\prime}$ is an increasing function of the frequency for $\omega \leq 0.01 \mathrm{~Hz}$ to become $\omega$ independent at higher frequencies. It is not likely that the observed "nonsol id" behavior is caused by wall slip, since the strain curve was a clear sine wave for all frequencies. Note that the plateau value of $\mathrm{G}^{\prime}$ is an increasing function of $\Phi$ for both dispersions and that for equal $\Phi L / D, G^{\prime}$ is higher for BA4 than for ASP5. 

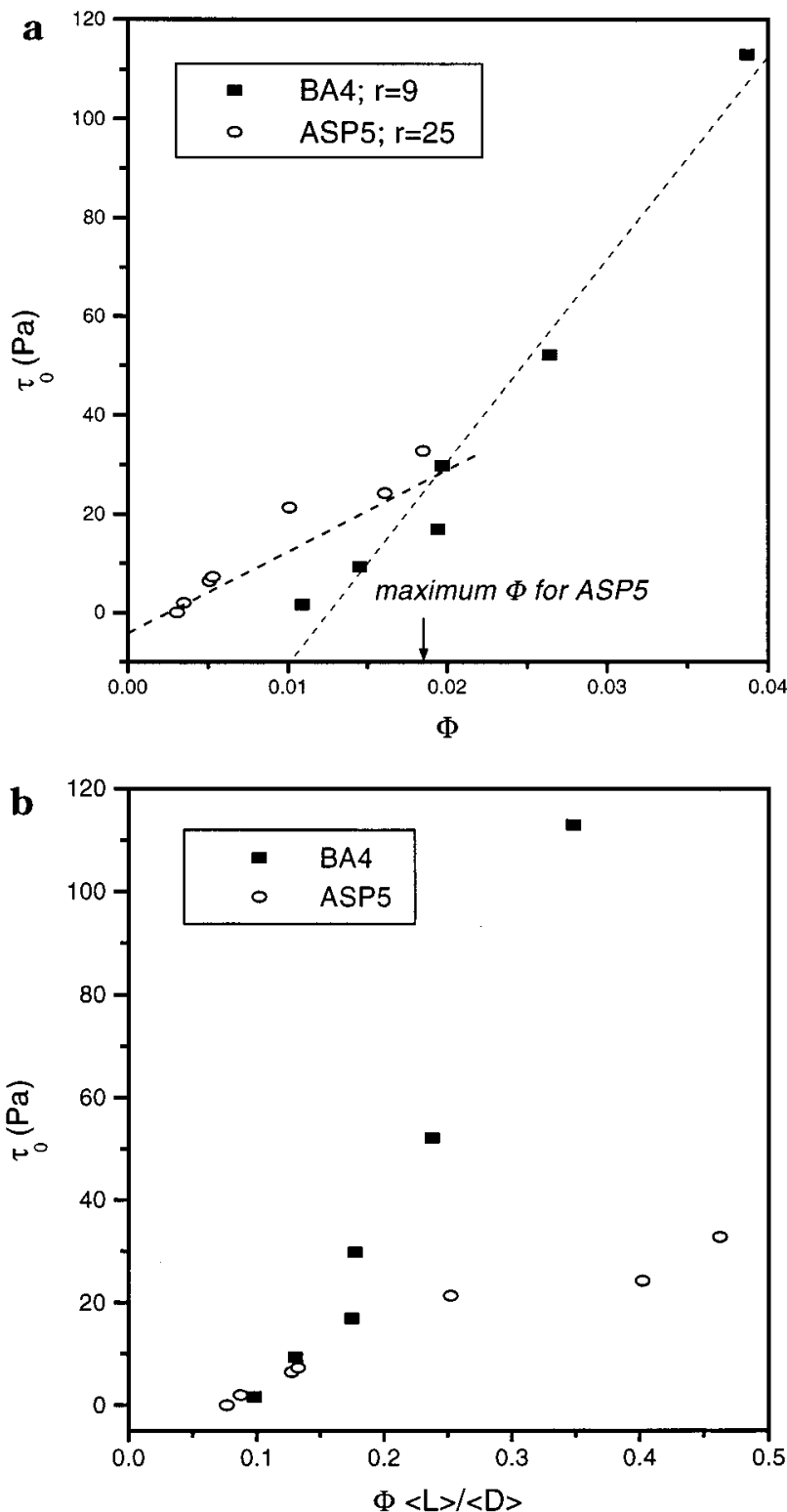

Figure 5. (a) Yield stress (as obtained by the vane method) as a function of the particle volume fraction for two different boehmite dispersions with $r=9$ (BA4) and $r=25$ (ASP5). The drawn lines are linear fits through the data points. (b) Same data as in part $a$, plotted against the product of $\Phi$ and the average aspect ratio $r=\langle L\rangle /\langle D\rangle$.

4.2. Boehmite Dispersions with Added LiCl. Appearance of Suspensions. Tosystematically study the changes in optical properties and colloidal stability of boehmite dispersions when lithium chloride is added, [ $\mathrm{LiCl}$ ] series of $0.17 \%, 0.33 \%, 0.6 \%$, and $1.15 \%$ ASP6 dispersions were prepared. The equilibration of the samples was slow, and during the first few weeks after preparation, changes still occurred in the appearance of the dispersions. Examples of equilibrated $\mathrm{LiCl}$ series for $\Phi=0.33 \%$ and $\Phi=1.15 \%$ are shown in Figures 7 and 8 . The samples shown on the photographs are respectively 2 weeks $(0.33 \%)$ and 6 weeks $(1.15 \%)$ old.

Figure 7 shows that, at equal ionic strength, the turbidity of the $1.15 \%$ samples is al ways higher than the turbidity of the $0.33 \%$ dispersions. The dispersions with $0<[\mathrm{LiCl}]<30 \mathrm{mM}$, remained fairly transparent, but small flocs were found to be formed in the $0.6 \%, 0.33 \%$ and $0.17 \%$ dispersions upon standing in glass tubes. Those flocs could not be redispersed by shaking the samples. A
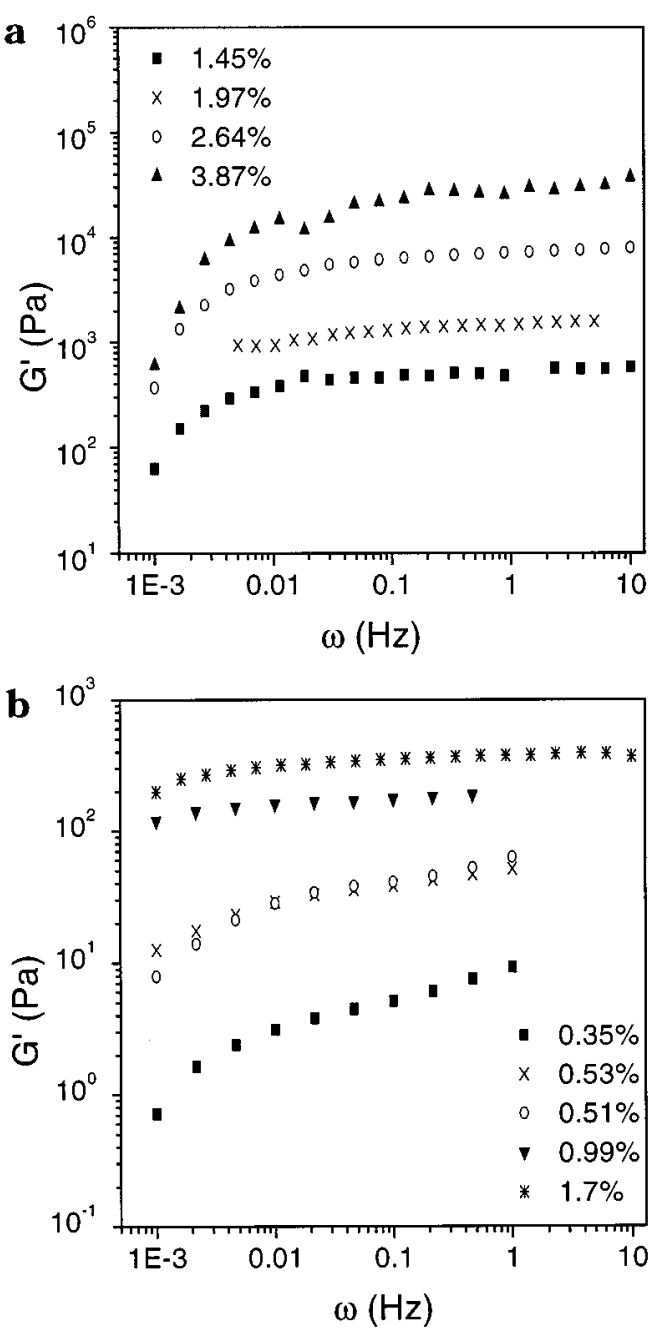

Figure 6. Frequency-storage modulus curves for dispersions of BA4 (a) and ASP5 (b) of several volume fractions.

drasticincrease of turbidity is found when [ $\mathrm{LiCl}$ ] is raised to concentrations higher than $10 \mathrm{mM}$. This turbid structure remains space filling up to $\mathrm{LiCl}$ concentrations of $75 \mathrm{mM}$, but in dispersions with higher $[\mathrm{LiCl}]$, syneresis occurs; thestrongly scattering structure only occupies the bottom phase of the dispersions, with a fairly clear supernatant. The volume of the bottom phase reduces significantly during the first 2 weeks after preparation of the samples, after which a "steady state volume" is reached. Those "separated"samples could behomogenized by shaking the tubes, but after a few days, the bottom phase had shrunk again to the same volume as before homogenizing. It is remarkable that the syneresis gives thesmallest bottom phasevolumes for thehighest particle volume fractions.

When the sample tubes are tilted $180^{\circ}$, a first observation about the strength of the structures within the dispersions can be made. In the $0.33 \%$ dispersions, selfsustaining structures are only formed in the LiCl-free sample and for $\mathrm{LiCl}$ concentrations between 20 and 100 $\mathrm{mM}$. In the $1.15 \%$ dispersions the structures are selfsustaining up to $[\mathrm{LiCl}] \approx 100 \mathrm{mM}$. For both $\Phi=0.33 \%$ and $\Phi=1.15 \%$ with $[\mathrm{LiCl}]=85 \mathrm{mM}$ this self-sustaining structure is not space-filling. The bottom layer of the samples with the highest $\mathrm{LiCl}$ concentrations $(\sim 0.5 \mathrm{M})$, slowly flows under gravity.

The occurrence of orientational ordering is observed by illuminating the dispersions between crossed polarizers as in Figure 8. Note that the vortex shape that is due to 

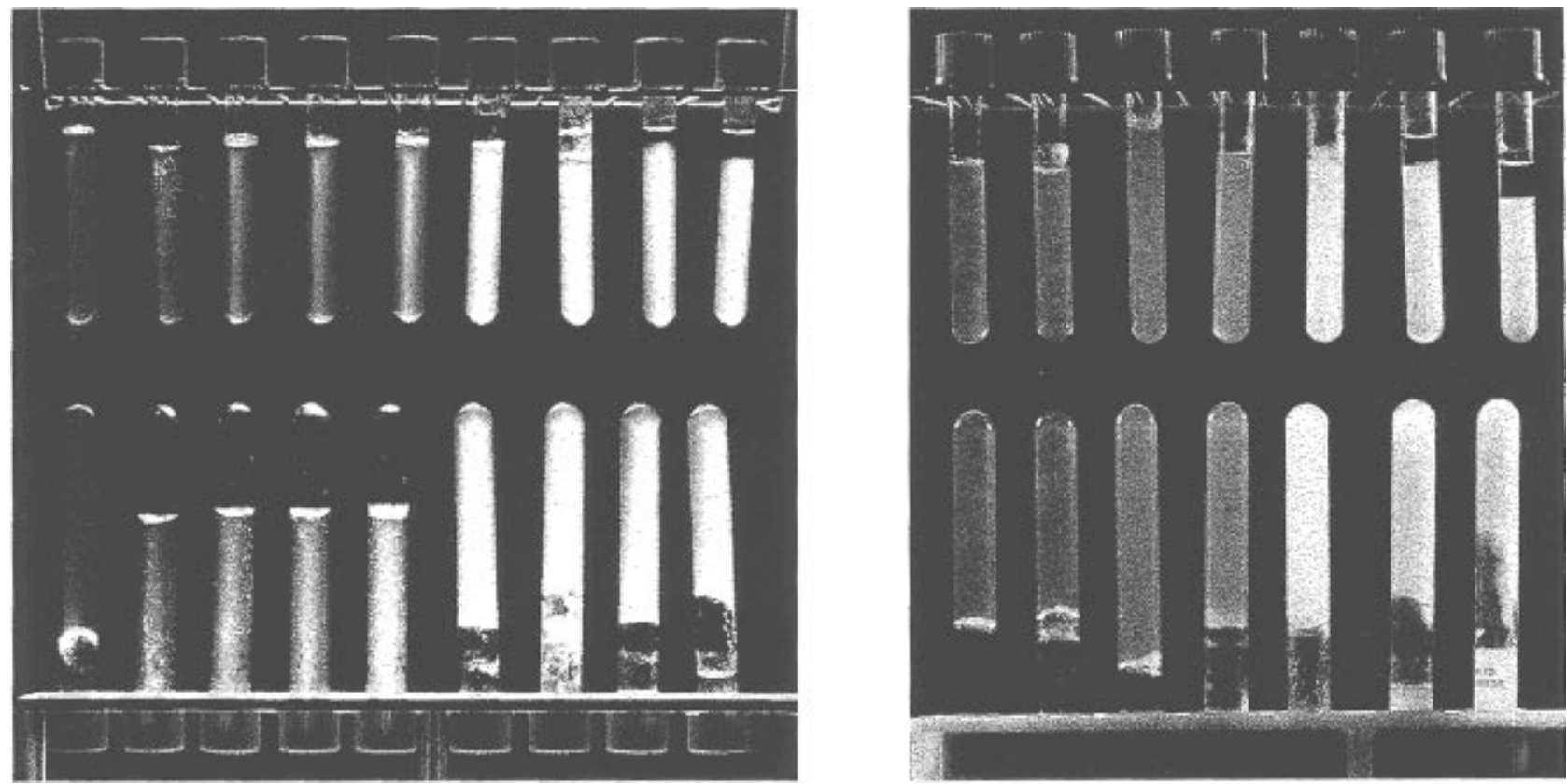

Figure 7. (a, left) Photograph of $\mathrm{LiCl}$ series of an ASP 6 dispersion of $0.33 \%$ with $0 \mathrm{mM}, 0.099 \mathrm{mM}, 0.55 \mathrm{mM}, 0.99 \mathrm{mM}, 10 \mathrm{mM}$, $29 \mathrm{mM}, 38 \mathrm{mM}, 92 \mathrm{mM}$, and $0.50 \mathrm{M} \mathrm{LiCl}$. In the tilted tubes subsequent decay and rise of the gel strength with increasing [LiCl] is demonstrated. (b, right) Photograph of $\mathrm{LiCl}$ series of an ASP6 dispersion of $1.15 \%$, from left to right: $0,0.4 \mathrm{mM}, 7.5 \mathrm{mM}, 49$ $\mathrm{mM}, 85 \mathrm{mM}, 0.47 \mathrm{M}$. F or this particle concentration, thestructures formed by the particles are self-sustaining for all but the highest $\mathrm{LiCl}$ concentration.
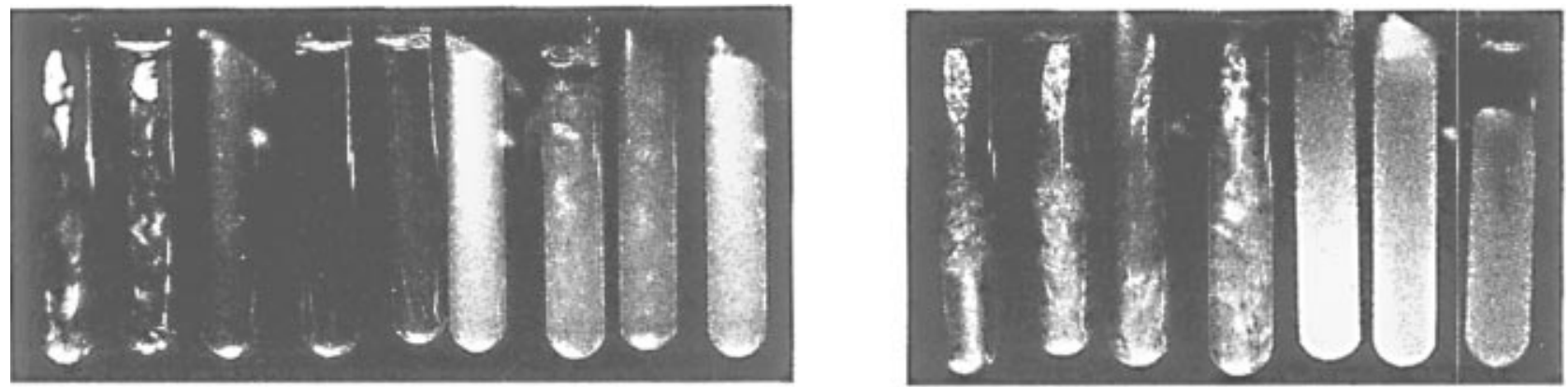

Figure 8. Photograph of the same tubes as in Figure 7, but now illuminated between crossed polarizers (a, (left), $\Phi=0.33 \%$; $b$ (right), $\Phi=1.15 \%$ ).

the mixing of the samples (weeks before the photographs were taken) is still visible in some of the dispersions. For $\mathrm{LiCl}$ concentrations bel ow $10 \mathrm{mM}$, the threshold for the formation of strongly scattering structures, the degree of ordering decreases for increasing $\mathrm{LiCl}$ concentration. In the dispersions with $\Phi=0.33 \%$, the bir efringence totally disappears for $\mathrm{c} \geq 0.1 \mathrm{mM}$. In the $1.15 \%$ dispersions, the birefringence remains visible in all dispersions with $\mathrm{LiCl}$ concentrations lower than $30 \mathrm{mM}$.

When more $\mathrm{LiCl}$ is added, in the dispersions with $\Phi=$ $0.33 \%$ birefringent patches appear for $[\mathrm{LiCl}]>30 \mathrm{mM}$, and birefringence is also visible on theedges of thesample with $\Phi=1.15 \%$ and $[\mathrm{LiCl}]=84 \mathrm{mM}$. The vortex shape of theordered domains suggests that theordering is frozen in during the sample preparation. When [ $\mathrm{LiCl}] \gg 100$ $\mathrm{mM}$, the birefringence totally disappears, and the dispersions form inhomogeneous bottom layer gels.

Static Light Scattering Measurements. Staticlight scattering (SLS) curves were measured of the $\mathrm{LiCl}$ concentration series of the four particle volume fractions of the ASP 6 dispersions. Due to the high turbi dity of the samples, nocurves could bemeasured for dispersions with $\Phi=0.6 \%$ and $1.15 \%$ for $\mathrm{LiCl}$ concentrations higher than $10 \mathrm{mM}$. The double logarithmic pl ot of the data points at scattering angles between $20^{\circ}$ and $60^{\circ}$ could be described

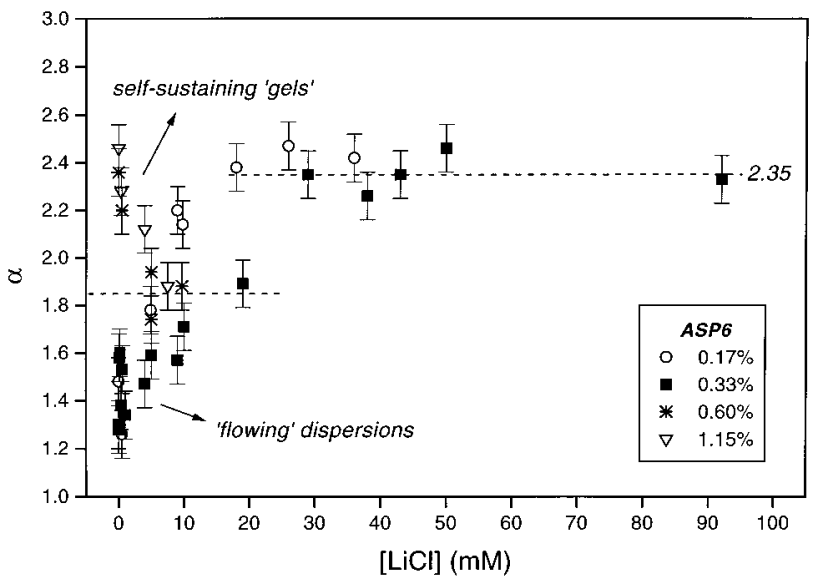

Figure 9. Negative slopes of the double-logarithmic plots of the SLS curves of ASP6. For networks with a self-similar structure, the magnitude of this negative slope may be interpreted as the (fractal) dimension of that structure.

by a linear fit. The negative slopes, $\alpha$, of those fits are shown in Figure 9. Note that upon addition of $\mathrm{LiCl}, \alpha$ increases for the two low volume fractions but decreases for the two higher vol umefractions. For $7.5 \mathrm{mM} \leq \mathrm{c}(\mathrm{LiCl})$ $\leq 20 \mathrm{mM}$ (that is below the threshold for formation of 

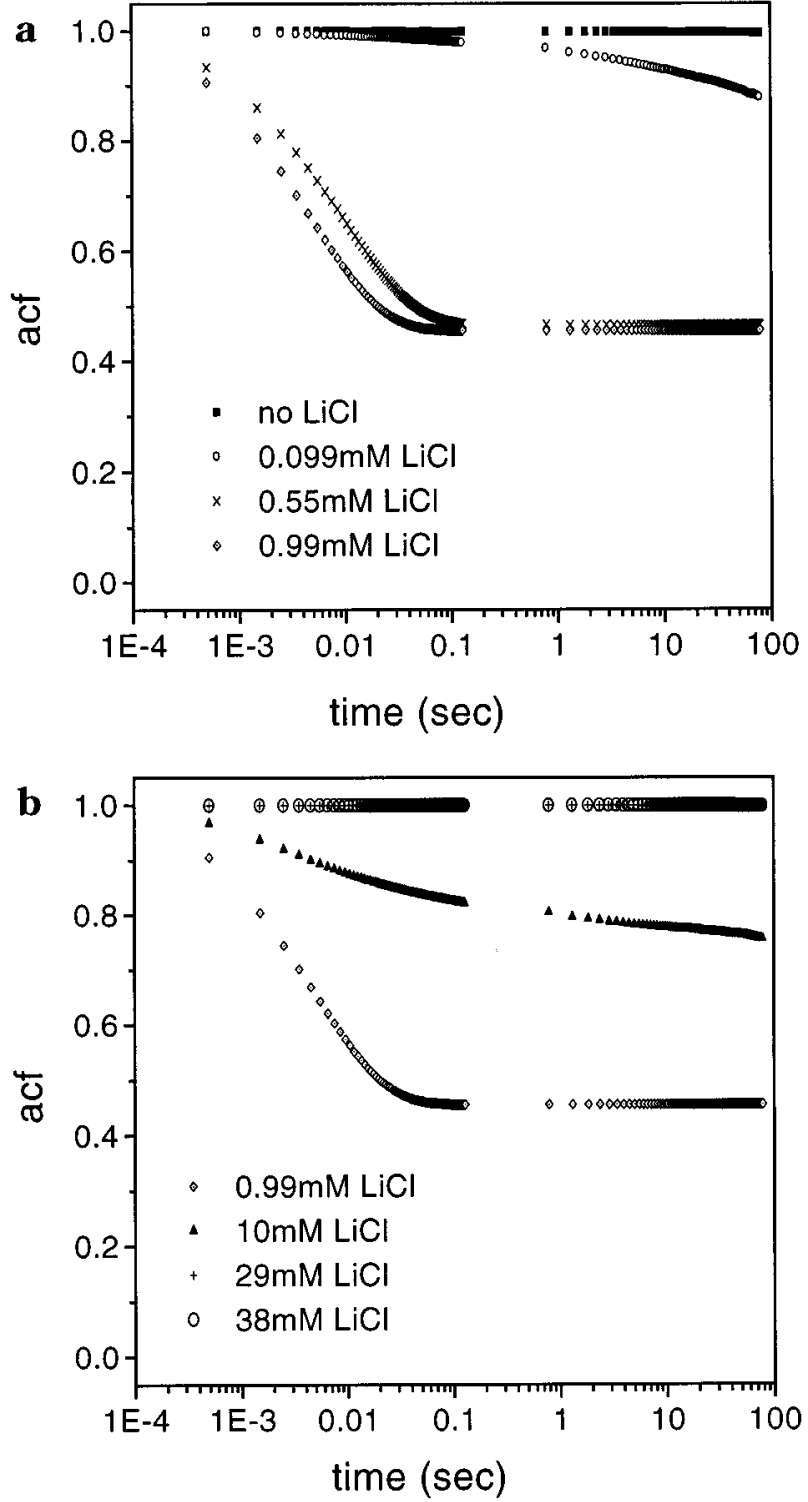

Figure 10. Decay of the intensity autocorrelation function as measured for ASP6-dispersions with $\Phi=0.33 \%$ at several lithium chloride concentrations. Part a shows the increase of the transl ational diffusion in regi on $\mathrm{I}$, and in part $b$, thefreezing of the particlediffusion in region II is shown. Theplateau value at autocorrelation function $\approx 0.5$ corresponds with a maximal displacement of $\sim 40 \mathrm{~nm}$.

strongly scattering structures), the negative slopes of all curves approach $\alpha=1.85$. When more $\mathrm{LiCl}$ is added to the low volume fraction dispersions, the negative slopes of all SLS curves approach, within experimental error, $\alpha$ $=2.35$.

DLS Measurements. The autocorrelation functions of $0.33 \%$ ASP 6 dispersions are given in Figure 10. Addition of $\mathrm{LiCl}$ first enhances the diffusion of the rods (Figure 10a), but at $\mathrm{LiCl}$ concentrations higher than 5.5 $\mathrm{mM}$, the diffusion rates reduce with increasing $[\mathrm{LiCl}]$ (Figure 10). Note that for $[\mathrm{LiCl}]=0.99 \mathrm{mM}$ the diffusion on short time scales is frozen (like for BA6 in Figure 4), whereas for $[\mathrm{LiCl}]=0.55,0.99$, and $10 \mathrm{mM}$ the autocorrelation function decays at short time scales but freezes for long time scales. It is striking that for none of the measured samples does the autocorrelation function fully relax within $80 \mathrm{~s}$.

Yield Stress Measurements(Vane). Theyield stress measurements wereperformed on a series of fivedifferent

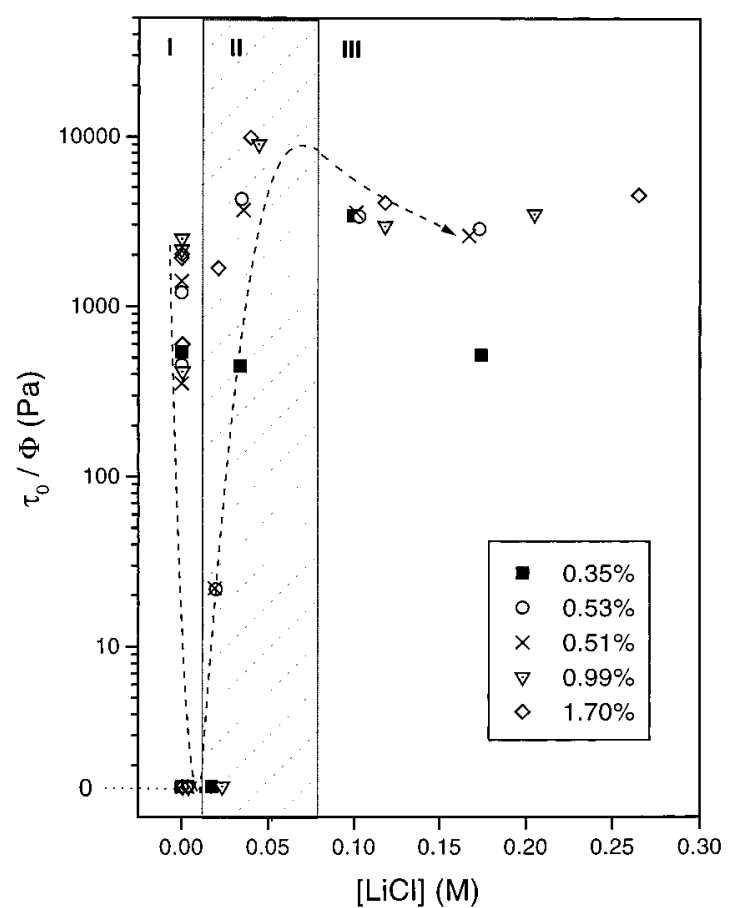

Figure 11. Yield stress per volume fraction for several ASP5 dispersions as a function of thelithium chloride concentration, measured with a Haake-vane rheometer at $0.2 \mathrm{rpm}$.

starting ASP 5 dispersions in which theionicstrength was increased every $24 \mathrm{~h}$. In Figure 11 the yield stress per volume fraction is plotted as a function of [ $\mathrm{LiCl}]$. The parameter $\tau_{0} / \Phi$ is related to the contribution of a single particle to the dispersion's yield stress.

With increase of the ionic strength of the dispersion, the "yield stress per particle" first decreases (in Figure 11 denoted as region I). For $10 \mathrm{mM} \leq[\mathrm{LiCl}] \leq 75 \mathrm{mM}$ (region II), the yield stress then steeply increases with [ $\mathrm{LiCl}]$ to reach a maximumvaluethat is higher than that measured in the dispersions without added salt for $[\mathrm{LiCl}] \approx 50 \mathrm{mM}$. In region III, the quotient $\tau_{0} / \Phi$ decreases again. I n contrast to the dialyzed samples, the vane readings for the dispersions with increased ionic strength $(>10 \mathrm{mM})$ showed a clear maximum, after which the torque on the vane decreased again. This indicates that the structure that causes the yield stress in the $\mathrm{LiCl}$ dispersions can indeed bebroken down by theshearing motion of thevanes.

The visual observations made of the tubes of ASP 6 (as in Figures 7 and 8) can well be compared with the yield stress curves of the ASP5 dispersions (Figure 11). In Figure 12 a "mechanical phase diagram" is drawn for the dispersions with $r \approx 25$ as a function of [ $\mathrm{LiCl}]$ and the particle volume fraction. The observations made on the ASP 6 samples with $\Phi=0.33 \%$ were used as a guide line to draw the dividing lines that mark thetransitions in the gel structure, because the phase behavior of the $0.33 \%$ dispersions as a function of [ $\mathrm{LiCl}]$ was studied in much moredetail than that for theother threevolume fractions. Also, the $[\mathrm{LiCl}]$ dependence of the phasebehavior as found for the samples with $0.17 \%, 0.6 \%$, and $1.15 \%$ did not conflict with that found for $\Phi=0.33 \%$.

\section{Discussion}

5.1. Boehmite Dispersions without Added Salt. Significance of Yield Stress Measurements. Our data show that onecan definea yiel d stress in aqueous boehmite dispersions (above a certain volumefraction). Thereality of the yield stress in those systems is supported by the 


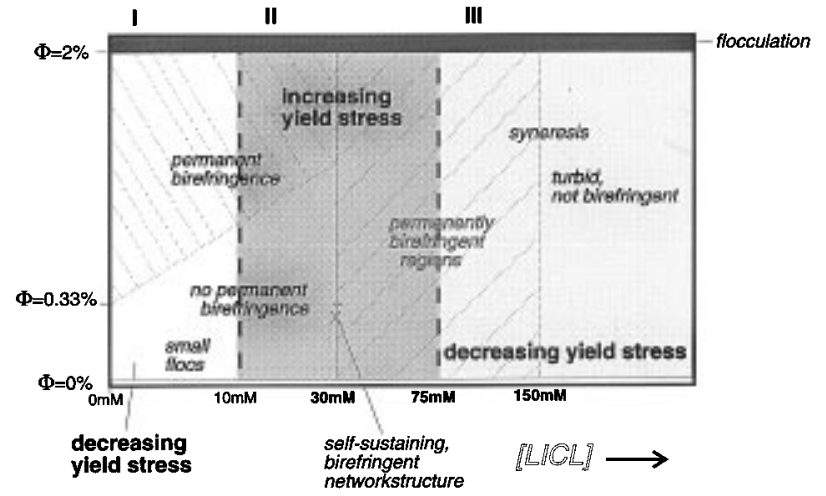

Figure 12. Combination of the rheology measurements (yield stress values) on ASP5 systems and visual observations of the ASP 6 dispersions shows that three $\mathrm{LiCl}$ concentration regions of different "gel-types" may be defined.

observation of self-sustaining gels. However, oscillatory shear measurements indicatethat on timescales of either $100 \mathrm{~s}$ (200 nm rods) and $10 \mathrm{~s}$ (90 nm rods) the yielding dispersions do fl ow (very slowly) when subjected to shear stresses smaller than the yield stress. Apparently, the structure causing the yielding behavior in the boehmite dispersions is not compl etel y rigid at shear stresses lower than the yield stress.

Nonetheless, the shear yield stress as obtained for our dispersions is a reproducible parameter and as such a good measureto quantify thestrength of thegel structure. Hence, straightforward measurement of the yield stress in a rotating vane geometry is a valuable tool for studies of gelling dispersions.

Relation between Structure Dynamics and Yielding Behavior. In the boehmite dispersions, yielding structures areformed abovea certain gel ling concentration $(0.35 \%$ for $r \approx 25$ and $1.5 \%$ for $r=9)$. The strength of these structures is reduced significantly when small amounts of $\mathrm{LiCl}(<10 \mathrm{mM})$ are added to the dispersions. F urthermore, the tor que on the vane is not reduced after reaching the maximum value, which we interpret as the yield stress. Apparently theyield stress is not caused by a network that can be broken down by theshearing motion of thevane. Thegelation in the dispersions without added salts is due to double layer repulsions. Yielding dispersions without added $\mathrm{LiCl}$ can thus be called "repulsive" gels in which therods aretrapped in "cages" of neighboring rods and double layers. In fact, a "repulsive gel" can be consi dered a colloidal glass, which has a very high viscosity at short time scales, due to the "freezing" of the rotational diffusion of the charged rods.

Figure 5b shows that the critical value of $\Phi\langle L\rangle /\langle D\rangle$ for the build-up of a yield stress is comparable for both types of boehmiterods and is of the order 0.08-0.09. Duetothe electric double layer inter actions this is much lower than the maximum packing fraction of a dispersion of hard rods, ${ }^{20}$ for which $\Phi L / D \approx 5$. Nonetheless, the gel strength of the dispersions of short rods (BA4) is a much steeper function of $\Phi\langle L\rangle /\langle D\rangle$ than that for ASP5.

It has been found that the gel strength in both types of dispersions increases with time, the rods (slowly) reorientate to the strongest yielding structure. Both the $\omega$-dependence of the storage modulus (Figure 6) and the decay of the autocorrelation function (Figure 4) show that the diffusion of the short rods (BA4 and BA6) is much faster than that of the longer rods (ASP5 and ASP6).

(20) Philipse, A. P. Langmuir 1996, 12, 1127, Corrigendum Langmuir 1996, 12, 5971.
Therefore, theshort rods will bemoreefficient in reaching the positions and orientations causing the highest yield stress than the long rods.

Thehighest gel strengths (actually low-shear viscosities) are measured in dispersions that do not show permanent birefringence. Accordingtothecalculations of Sparnaay, ${ }^{21}$ the repulsive interaction energy of two cylinders is maximal for parallel alignment. Buining ${ }^{17}$ showed that the structure of the birefringent boehmite dispersions consists of a limited number of domains in which the rods show orientational ordering. In a shear field, each domain may flow as a single "packet" of rods. Probably, the shear stress needed to translate domains with respect to each other is small compared to the shear stress necessary to movea far higher number of indi vidual, "caged" particles. This shows that for rod gels, the yield stress is not simply related to the product of the number density and the average interaction potential but also depends on the microstructure of the gel.

When the ASP5 and ASP 6 dispersions were concentrated to particle volume fractions higher than $2 \%$, irreversible flocculation occurred. Apparently, for such high particle concentrations, the overlap of double layers flattens out the interaction potential to such extent that the repulsion barrier for fl occulation becomes ineffective.

5.2. Boehmite Dispersions with Added LiCl. Interaction Energy Calculations. Theaddition of $\mathrm{LiCl}$ to the boehmite dispersions results in changes in the rod interactions. The interaction energy between boehmite rods (Hamaker constant, ${ }^{16} \mathrm{~A}=6 \times 10^{-20} \mathrm{~J}$ ) as calculated using the expressions of Sparnaay ${ }^{21}$ for the electrostatic repulsions and the van der Waals attractions between twocylinders is shown in Figure 13 for twoionicstrengths. The calculations predict colloidal stability for smooth cylinders with $\mathrm{L}=180 \mathrm{~nm}, \mathrm{D}=8 \mathrm{~nm}$, and $\xi=70 \mathrm{mV}$ (comparable to the "average" of ASP5 and ASP6) at monovalent electrolyteconcentrations as high as $500 \mathrm{mM}$.

Nonetheless, Figure 13 shows that the interaction potential is very sensitive to variation of particle dimensions and surface potential and rod orientation. The boehmite rods in this work are not smooth cylinders but have irregular surfaces and show a significant polydispersity in particle dimensions. For different parts of a single rod or different rods, surfacechemistry details may also vary, causing different isoelectric points and surface potentials. Two thin, weakly charged parts of rods that approach each other in crossed orientation will aggregate at much lower ionic strength than two thick, well-charged parts of rods approaching in parallel orientation. Due to such polydispersity in interactions not all rods will become unstable at the same ionic strength, and the number of flocculated rods will be an increasing function of the electrolyte concentration.

Structure, Dynamics, and Yield Stress in the Three [LiCI] Regions. When gelled boehmite dispersions are brought to different $\mathrm{LiCl}$ concentrations, effectively three different regimes can be observed. In Figures 11 and 12 the different regimes are indicated by $\mathrm{I}, \mathrm{II}$, and III, and the yield stress in the different regi mes is subsequently a decreasing, rising, and again decreasing function of the $\mathrm{LiCl}$ concentration. A similar rise-anddecay of the gel strength upon addition of electrolyte are madein dispersions of bentonite. ${ }^{2}$ Darley ${ }^{2}$ interprets this peculiar behavior in terms of differences between "flocculation" in the secondary mi nimum and "aggregation" in the primary minimum. No secondary minimum aggregation is to be expected for boehmite rods (Figure 13) so

(21) Sparnaay, M. J . Recl. Trav. Chim. Pays-Bas. 1959, 78, 680. 

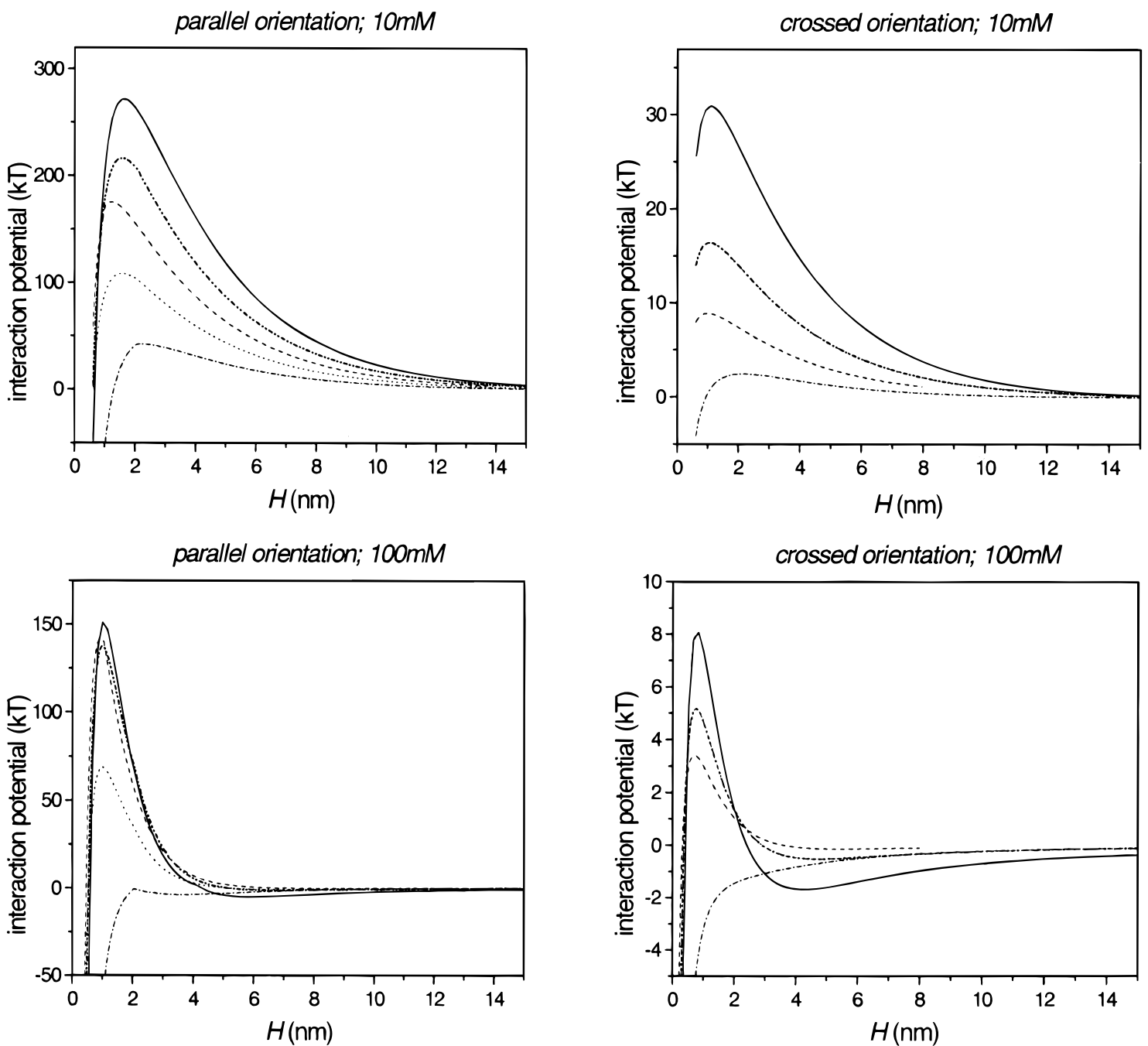

Figure 13. Interaction potential of two cylinders with the Hamaker constant of boehmite in water: $-, \zeta=70 \mathrm{mV}, \mathrm{L}=180 \mathrm{~nm}$, $\mathrm{D}=8 \mathrm{~nm} ; \cdots, \zeta=70 \mathrm{mV}, \mathrm{L}=180 \mathrm{~nm}, \mathrm{D}=16 \mathrm{~nm} ;---, \xi=70 \mathrm{mV}, \mathrm{L}=90 \mathrm{~nm}, \mathrm{D}=8 \mathrm{~nm} ;-\cdot-, \zeta=70 \mathrm{mV}, \mathrm{L}=80 \mathrm{~nm}, \mathrm{D}$ $=4 \mathrm{~nm} ;-\cdots-, \zeta=35 \mathrm{mV}, \mathrm{L}=180 \mathrm{~nm}, \mathrm{D}=8 \mathrm{~nm}$.

Darley's model is not applicablefor our dispersions. Here, we will attempt to interpret the relation between the electrolyte concentration and the gel properties of our boehmite dispersions in terms of polydispersity in particle interactions.

Region I: $\mathbf{0}<$ [LiCl] $<\mathbf{1 0} \mathbf{~ m M . ~ I n ~ t h e ~ f i r s t ~ [ ~} \mathrm{LiCl}]$ range, the yield stress decreases (Figure 11) and the dynamics of the rods become less restricted (Figure 10) with increasing $\mathrm{LiCl}$ concentration. In a dispersion with only monovalent ions, ${ }^{22}$ the Debye length $\kappa^{-1}$ depends strongly on the ionic strength $\mathrm{c}(\mathrm{mol} / \mathrm{l})$

$$
\kappa^{-1}=0.304 / \sqrt{\mathrm{c}} \mathrm{nm}
$$

Upon addition of small amounts of $\mathrm{LiCl}$, the doublelayers are thus severely compressed, and the entanglement of the rods is reduced. Figure 10 shows that the diffusion coefficient is the steepest function of $[\mathrm{LiCl}]$ for the lowest $\mathrm{LiCl}$ concentration; the difference between the curves of $[\mathrm{LiCl}]=0.099 \mathrm{mM}$ and $[\mathrm{LiCl}]=0.55 \mathrm{mM}$ is much greater than that between $[\mathrm{LiCl}]=0.55 \mathrm{mM}$ and $[\mathrm{LiCl}]=0.99$ $\mathrm{mM}$. Notethat theautocorrel ation function does still not fully decay on long time scales for the dispersions with 0.55 and $0.99 \mathrm{mM} \mathrm{LiCl}$. Hence, even when the double

(22) I sraelachvili, J. N. Intermolecular and Surface Forces, with Applications to Colloidal and Biological Systems; Academic Press: London, 1985. layer interactions are sufficiently screened to render the dispersions flowing, the suspended particles hinder each other's diffusion significantly.

Small flocs were observed in the flowing boehmite dispersions at these low ionic strengths, but not in the self-sustaining samples. In other work, we report the orthokinetic flocculation of similar boehmite rods $(r=22)$ for $[\mathrm{LiCl}] \geq 0.2 \mathrm{mM}$, that involved only a small fraction of the rods. These observations are in agreement with the concept of "polydispersity of interactions". When the ionic strength of a boehmite dispersion is increased but still lower than $10 \mathrm{mM}$, only the least stable (thin, short, ill-charged) rods will aggregate. In a gelled dispersion, wherethe particledynamics arefrozen in, the probability that two "attractive" particles meet is practically negl $i$ gible.

Region II: 10mM $<$ [LiCl] $\leq 75 \mathrm{mM}$. In the shaded region of Figure 11, the yield stress of ASP5 dispersions increases with rising $\mathrm{LiCl}$ concentration and reaches a maximum for $[\mathrm{LiCl}] \approx 50 \mathrm{mM}$, at which the yield stress is much higher than that in thedispersions without added $\mathrm{LiCl}$. Figure $10 \mathrm{~b}$ shows that in region II the dynamics of the ASP6 rods freeze with increasing $[\mathrm{LiCl}]$. A large enough fraction of the rods has become attractive to form a space-filling network that causes a yield stress. The decrease of the vane reading after reaching its maximum 
value confirms the existence of interparticle bonds that are broken by the shearing motion of the vane.

Probably, the particlestructurein such a network may be considered as a skeleton of flocculated particles in a dispersion of stable rods. The gel strength is caused by a combination of thenetwork and the high viscosity of the "interspacing" suspension. Thefrozen birefringent regions in those dispersions demonstrate both the presence of nonaggregated rods and the slowness of the particle dynamics in those dispersions.

In I it is estimated that in a homogeneous network the density is at least $\Phi\langle\mathrm{L}\rangle\langle\mathrm{D}\rangle=0.7$. F or the boehmite rods, the highest concentration $(\Phi=0.33 \%$ ) corresponds with $\Phi\langle\mathrm{L}\rangle\langle\mathrm{D}\rangle \approx 0.09$. Apparently, the network formed in the boehmite dispersions has a heterogeneous structure. Figure 9 shows that the dimension of the structure in flocculating boehmite dispersions is smaller than 3 (d = 2.35) and independent of the particle and electrolyte concentration for $0.17 \% \leq \Phi \leq 0.33 \%$ and $25 \mathrm{mM} \leq[\mathrm{LiCl}]$ $\leq 100 \mathrm{mM}$. At those concentrations, the structure of the network in the gels is self-similar over long ranges in the gel, and probably mainly determined by aggregation kinetics. ${ }^{12}$ Possibly networks formed in dispersions with higher volume fractions are the same. This speculation could not bechecked, as thehigh turbidity of thosesamples prohibited SLS measurements.

The dimension of the network formed by the boehmite rods is much higher than $\mathrm{d} \approx 1.95$ as found by Brunner et al. ${ }^{23}$ for networks of iron hydroxide rods. Apparently, the flocculation rate in the iron hydroxide dispersions is much higher than in the boehmite dispersions, yielding a network structure of lower density. Thelow flocculation rate of boehmiteis probably related to the "polydispersity in interactions": not every collision between pairs of rods will lead toaggregation, and theaggregation may becalled "reaction limited". Micrographs of the iron hydroxide particles do not show distinct surface inhomogeneities, and all rods will probably become attractive as soon as the ionic strength is high enough to screen the double layer repulsion. Above that "flocculation threshold" the aggregation is "diffusion limited". Note that the dimensions of the rod networks are in reasonable agreement with the dimensions reported for either diffusion limited cluster aggregation $(\mathrm{d}=1.85)$ and reaction limited cluster aggregation $(d=2.1)$ in spheredispersions. ${ }^{12}$ (This point is discussed in I.)

For $\mathrm{c}<30 \mathrm{mM}$, no indications of the formation of a space-filling cluster network exist, sohere $\alpha$, the negative slope of theSLS curvecannot beinterpreted as a network dimension.

Region III: [ $\mathrm{LiCl]} \geq \mathbf{7 5} \mathrm{mM}$. In region III of the "mechanical phase diagram" in Figure 12 the network structure formed by the aggregating particles shrink and expel water. The vane measurements reveal that even before this syneresis occurs, the yield stress of samples with high $[\mathrm{LiCl}]$ is reduced significantly with respect to the maximum values. Apparently the boehmite network is space-filling up to $[\mathrm{LiCl}] \approx 50 \mathrm{mM}$ but collapsesat higher ionic strength.

With increasing $\mathrm{LiCl}$ concentration, a larger fraction of the rods flocculates; this is confirmed by the absence of birefringent regions in the dispersions with $[\mathrm{LiCl}]>100$ $\mathrm{mM}$. When a large fraction of the rods flocculates, the particle concentration and thus the viscosity of the liquid phase between thenetwork branches reduce. At thesame time, more flocculated particles will try to reduce their 153.

(23) Brunner, R.; Gall, S.; Wilke, W.; Zrinyi, M. Physica A 1995, 214,

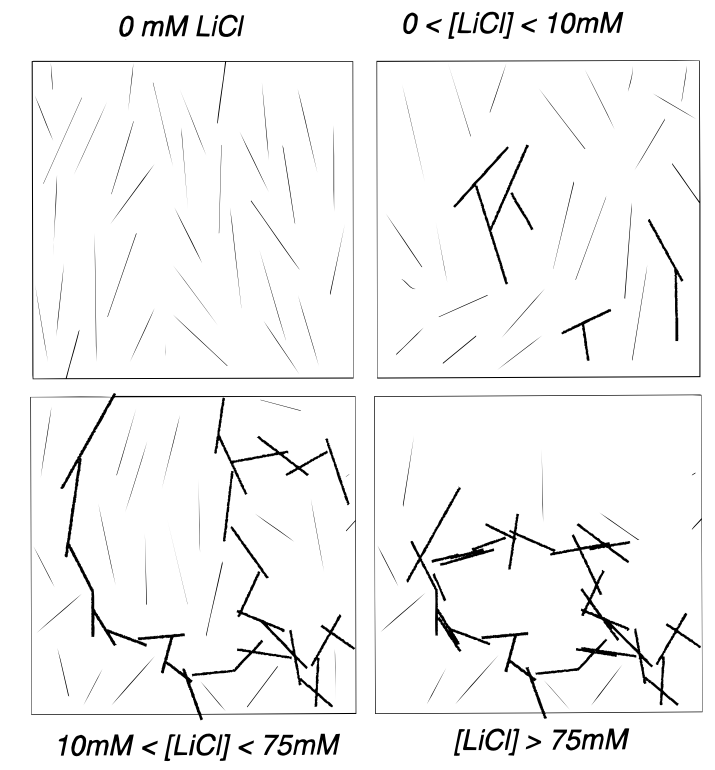

Figure 14. Schematic drawing of the particle structure in boehmite dispersions with different $\mathrm{LiCl}$ concentrations.

intersurface distance (Figure 14). Rods that initially aggregated in perpendicular orientation may rotatetoform a more stable parallel bonding. Due to such processes water will be expelled from the network structure (syneresis), and inhomogeneous structures with a reduced gelstrength will be formed. Note that the formation of paral lel aggregates al so effectivel y reduces the number of links in the network and so decreases the yield stress.

The syneresis is the fastest and the network volume the smallest for the gels with the highest rod concentrations. The higher mass density of the network branches in a gel of higher rod concentration may enhancea collapse under gravity. Furthermore, a higher degree of branching (favored by the high rod concentration) may stimulate syneresis by reorientation of particles.

This decrease of the network strength with a growing number of flocculated particles and increasing particle attractions again shows that the strength of a rod gel cannot simply be estimated from the product of the interaction potential and the number of interparticle bonds.

\section{Summary and Conclusions}

We have studied the gelation in aqueous boehmite dispersions with two different aspect ratios as a function of the particle and lithium chloride concentration.

The vane rheometer proves to be an adequate tool to measure the gel strength of those dispersions. Supplementary low-frequency oscillatory shear measurements are valuable to study the yielding behavior on long time scales.

At low ionic strength, the gelation in boehmite gels is caused by "freezing" of the rotational diffusion of the rods as a result of double layer overlap. The diffusion of the rods in those gels, however, may still be significant on long times. In fact, theserepulsive gel s can beconsidered as colloidal glasses with a very high low-shear viscosity.

Due to polydispersity in particle interactions, caused by surface inhomogeneities, irregul arities in shape and a reasonable spread in the particle dimensions, some rods flocculateat considerably lower ionicstrength than others, and the number of colloidally unstable particles is an increasing number of the $\mathrm{LiCl}$ concentration. 
In dispersions with added $\mathrm{LiCl}$, three different types of behavior can be distinguished. In region I ([LiCl] $<10$ $\mathrm{mM}$ ), the dominant particle interactions are repulsive. With an increase of the ionic strength, the double layer interactions are reduced, causing a break-up of the gels and the formation of some flocs. In region II $(10 \mathrm{mM}<$ $[\mathrm{LiCl}]<75 \mathrm{mM}$ ), there is a balance between repulsive and attractive interactions. As soon as the number of flocculated rods is high enough to form a space-filling structure, a new gel is formed, consisting of a network of aggregated particles in a dispersion of stable rods. Because not all rod pairs form clusters, the aggregation kinetics is slower than diffusion limited, yiel ding a network dimension $d \approx 2.35$. Further increase of theionicstrength (region III, [LiCl] > $75 \mathrm{mM}$ ) renders the behavior dominated by particle attractions. When a largefraction of theparticles aggregates, theviscosity of the continuous phase is decreased, which reduces the network strength. Reorientation of rods in clusters to form parallel aggregates then induces a denser network structure, causing syneresis and a decrease of the yield stress.

This work shows that for both repulsive and attractive rod gels, theyield stress is not simply related totheproduct of interacting particles and the strength of theinteraction potential. Parallel alignment in either an ordered domain or a cluster increases the interaction strength per rod pair but reduces the yield stress since the packets of aligned rods do not have to be broken to liquefy the dispersions.

Acknowledgment. We thank Dr. J . K. G. Dhont for his help with the DLS experiments and interpretation, M. D. Green and N. Pashias (University of Mel bourne) for their assistance with the vane experiments, and $M$. van Bruggen for providing the program for the interaction potential calculations. This work was supported by The Netherlands Organization for Chemical Research (SON) with financial aid from The Netherlands Organization for Scientific Research (NWO). A.W. thanks theAdvanced Mineral Products Center for financial support and hospitality during her stay in M el bourne, where part of this work was done.

LA970376Z 